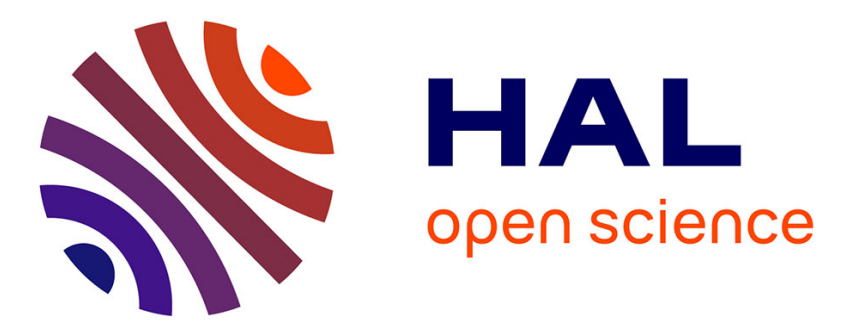

\title{
Automatic Optimal Input Command for Linearization of cMUT Output by a Temporal Target
}

Sébastien Ménigot, Dominique Certon, Dominique Gross, Jean-Marc Girault

\section{To cite this version:}

Sébastien Ménigot, Dominique Certon, Dominique Gross, Jean-Marc Girault. Automatic Optimal Input Command for Linearization of cMUT Output by a Temporal Target. IEEE Transactions on Ultrasonics, Ferroelectrics and Frequency Control, 2014, 61 (10), pp.1742-1753. 10.1109/TUFFC.2013.006330 . hal-01069932

\section{HAL Id: hal-01069932 https://hal.science/hal-01069932}

Submitted on 30 Sep 2014

HAL is a multi-disciplinary open access archive for the deposit and dissemination of scientific research documents, whether they are published or not. The documents may come from teaching and research institutions in France or abroad, or from public or private research centers.
L'archive ouverte pluridisciplinaire HAL, est destinée au dépôt et à la diffusion de documents scientifiques de niveau recherche, publiés ou non, émanant des établissements d'enseignement et de recherche français ou étrangers, des laboratoires publics ou privés. 


\title{
Automatic Optimal Input Command for Linearization of cMUT Output by a Temporal Target
}

\author{
Sébastien Ménigot, Dominique Certon, Dominique Gross, and Jean-Marc Girault
}

\begin{abstract}
Capacitive micromachined ultrasonic transducers (cMUT) are a promising alternative to the piezoelectric transducer. However, their native nonlinear behaviour is a limitation for their use in medical ultrasound applications. Several methods based on the precompensation of a preselected input voltage have been proposed to cancel out the harmonic components generated. Unfortunately, these existing pre-compensation methods have two major flaws. The first is that the pre-compensation procedure is not generally automatic, and the second is that they can only reduce the second harmonic component. This can therefore limit their use for some imaging methods which require a broader bandwidth, e.g. to receive the third harmonic component. In this study, we generalized the pre-setting methods in order to reduce all nonlinearities in the cMUT output. Our automatic pre-compensation method can work whatever the excitation waveform. The pre-compensation method is based on the nonlinear modelling of harmonic components from a Volterra decomposition where the parameters are evaluated by using a Nelder-Mead algorithm. To validate the feasibility of this approach, the method was applied to an element of a linear array with several types of excitation often encountered in encoded ultrasound imaging. The results showed that the nonlinear components of the mechanical displacement were reduced by up to $21.2 \mathrm{~dB}$.
\end{abstract}

Index Terms

Adaptive system, closed loop system, input voltage, optimal control, optimization, pre-compensation, signal processing, cMUT.

\section{INTRODUCTION}

O VER the last fifteen years, several alternatives to traditional piezoelectricity technology have emerged. Among the most promising are cMUTs (capacitive micromachined ultrasonic transducers) [1]. Pressure generation results from movements of a few hundred micro-membranes collectively actuated by dynamic electrostatic forces. They offer many advantages, including direct integration of the preamplifying electronics in the head probe [2], greater acoustic efficiency and broader bandwidth [3] than their counterpart piezoelectric-based transducers.

Although many applications have demonstrated the feasibility of cMUT probes [4], [5], their native nonlinear behaviour [6] (due to the electrostatic forces) requires the use of non-standard waveforms as input signals to produce an output pressure field with the desired shape. This problem has already been addressed for two medical imaging applications for which nonlinearity of cMUTs would be a severe drawback, i.e. tissue harmonic imaging [7] and ultrasound therapy [8]. Tissue harmonic imaging aims to image the nonlinear properties of tissues. Contrast improvement is made possible by either filtering the harmonic components of the backscattered signal, or by using specific encoded excitation signals to reinforce the harmonic extraction, e.g. the pulse inversion technique [9]. Nevertheless, the cMUT nonlinearity mixes with that originating from the medium, leading to contrast reduction in the ultrasound image and cancelling out of encoding advantages. The value of therapeutic ultrasound has been demonstrated over nearly seventy years [8]. The efficiency of applications using cMUTs has recently been demonstrated [10] since a pressure level of around $1 \mathrm{MPa}$ can be delivered by the probe. However, clinical applications require better control of the output pressure field, with a low level of harmonic generation. These harmonic components can create local heating inside the housing lens, with the risk of untimely damage to the probe. Moreover, taking into account the diffraction properties, the harmonic components focus on a smaller focal spot than that expected with the fundamental component, with a risk of acoustic overload and local sonoporation. These two applications have one common point for transducer use, both require low bandwidth signal excitation, i.e. with a bandwidth lower than $40 \%$. However, the need to control the shape of the output pressure field emitted by a cMUT-based probe is not restricted to these applications but involves a wide range of new medical imaging methods requiring broad bandwidth excitation signals, such as encoded imaging and imaging of high order harmonics [11]. In order to take advantage of cMUT technology to improve the performance of such imaging modalities, effective automatic control methods must be developed to control the waveforms emitted by a cMUT-based transducer.

Sébastien Ménigot is with IUT Ville d'Avray, Université Paris Ouest Nantere La Défense, Ville d'Avray, France. Jean-Marc Girault and he are also with Université François-Rabelais de Tours, Inserm, Imagerie et Cerveau UMR U930, Tours, France.

Dominique Gross and Dominique Certon are with Greman, UMR 7347, Université François-Rabelais de Tours, Tours, France.

E-mail: sebastien.menigot@univ-tours.fr, jean-marc.girault@univ-tours.fr, dominique.gross@univ-tours.fr, dominique.certon@uni-tours.fr.

Manuscript received July 19, 2013; revised September 30, 2014. 
Two types of approach are currently available in the literature to control the output waveform of a cMUT. The first is based on forward electrical analysis of its behaviour. Using an equivalent electro-acoustic circuit describing the cMUT, Oguz et al. [12] established nonlinear analytical relationships between input electrical degrees of freedom and output acoustic degrees of freedom. These relationships could then be inverted to find analytically the input voltage which produced the targeted pressure. Although this provides a physical understanding of the compensation, this solution requires input simulation data which are not always known to the users. Satir and Degertekin [13] have proposed adding a passive component (resistance, capacitance or inductance) in series with the cMUT, in order to convert the input voltage control into an input charge control as a technique to control mechanical displacement of electrostatic actuator displacement [14]. In other words, the circuit made with the added passive impedance and the cMUT has the role of a nonlinear filter whose gain is automatically controlled: the increase in the snapshop voltage applied to the cMUT leads to the increase in the static capacitance and hence the reduction in circuit gain (the higher the voltage applied the lower the circuit gain). Since the passive component can be placed in the probe head, this technique is invisible to the user. However, it targets only low bandwidth signals. Moreover, when series resistance or capacitance is used, the voltage amplitude required to drive the transducer can be dramatically increased. The most promising solution is probably that based on inductance. The second type of approach, known as the input shaping method, acts directly on the shape of the input electrical voltage and is intended to reduce the level of harmonic components of the pressure spectrum emitted. Zhou et al. [15] developed an approach based on the principle of the Wiener filter in order to suppress the dependence of the square voltage in the electrostatic force. The same technique was then extended to reduce acoustic crosstalk in $1 \mathrm{D}$ linear array [16]. Despite offering a level of efficiency, the resolving method can be problematic in the case of singularity, since there is no regularization. The method proposed by Novell et al. [17] starts with a sine-shape excitation waveform at a given frequency $f_{0}$ and adds a component at $3 f_{0}$ whose amplitude and phase are empirically tuned in order to cancel out the second harmonic. This method resolves the drawback of the first but requires fine and difficult calibration which could limit its routine use. Moreover, although it has been successfully applied to pulse inversion imaging [18], it generates third harmonic components that can create additional problems, as already discussed for therapy. Finally, none of these methods are generally automatic and they are often limited to reduction of only the second harmonic.

The aim of this study was therefore to develop an automatic method of waveform optimization in order to suppress nonlinear components generated by cMUT. The method, called adaptive optimal command [19], is therefore an extension of the approach proposed by Novell et al. [17] where the input signal is modified using a nonlinear filtering technique [20] rather than manually adding together high order harmonic components. The method is thus applicable to a wide range of input waveforms and not restricted to sine-shape only. We therefore modified and adapted the linearization by Volterra series for loudspeakers [21] to the cMUT. Nonlinear filtering parameters of the input signal are automatically sought so that the output waveform can target the desired waveform. This approach amounts to identifying the cMUT as a nonlinear block model whose parameters are automatically fitted by adding a closed loop to the cMUT. This optimal command method can be directly applicable to any cMUT, since no a priori information about the cMUT is required.

The main challenges to achieving this aim are first to implement an automatic procedure for determining the desired waveform consistent with the physical properties of the cMUT, and secondly to find the optimal parameters of the nonlinear filter automatically. To validate the concept of adaptive optimal command, we show here experimental validation of the method based on measurements of displacements (obtained with laser interferometer) collected at the surface of a cMUT-based medical linear array immersed in water. The experimental measurements involved low bandwidth signal excitation (tissue harmonic imaging or therapy) for application of harmonic imaging methods or therapeutic methods. Moreover, the efficiency of our technique was also demonstrated for applications requiring a signal with $100 \%$ fractional bandwidth.

\section{Automatic Selection of the cMut Output Signal Desired}

Automatic determination of the desired output signal is a key step in our method since, as indicated above, it consists of finding desired output signals consistent with cMUT performance, i.e. in terms of peak-to-peak amplitude. Fig. II shows how the automatic determination worked using the cMUT in open loop. Since the aim is to find the cMUT output signal without harmonic components, the target signal $c(n)$ has to be composed of the single fundamental component. This targeted signal is therefore extracted by filtering the cMUT output signal when the cMUT input signal is the non-compensated signal $u(n)$.

The procedure is as follows. A usual input signal $u(n)$, with shape and spectral properties in agreement with the application, is applied to the cMUT input. The amplitude of the usual non-compensated input signal $u(n)$ is set to avoid the collapse event [22]. Note that the collapse phenomenon, also called pull-in, is the situation where electrostatic forces applied to the membrane are not balanced by elastic return forces, meaning that the membrane collapses and touches the bottom of the cavity.

The output of the transducer $y(n)$ can be either pressure field or mechanical displacement. Without compensation, its spectrum contains both linear and nonlinear components. In parallel with the cMUT block, an optimal moving average filter (MA filter) is applied to identify the linear component of the cMUT output signal without compensation. It provides the target output signal $c(n)$ with the same output amplitude as a linear component of the output signal $y(n)$, but its spectrum contains only linear components. Starting from the linear input-output relationships of the MA filter expressed under matrix form, the 


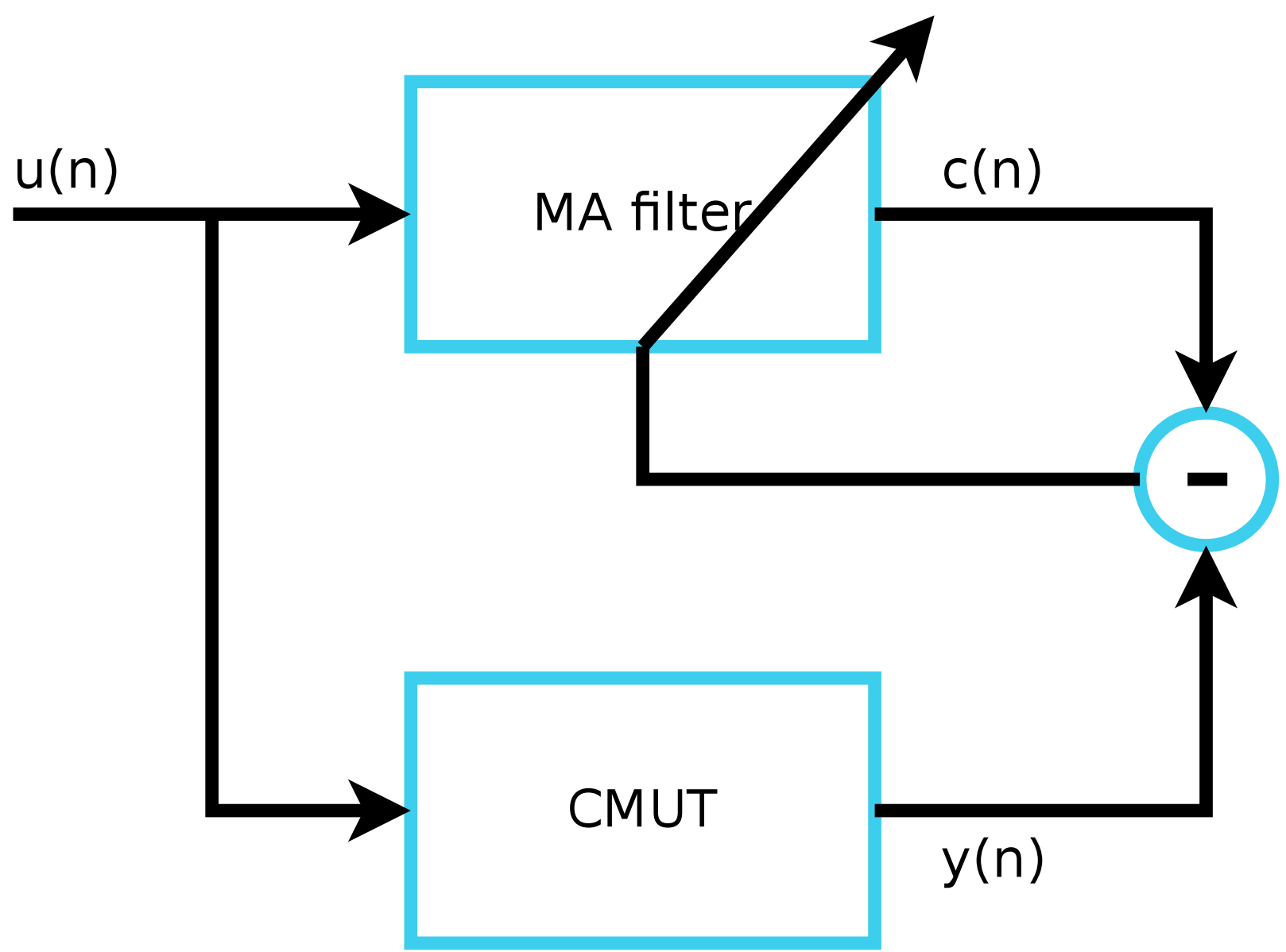

Fig. 1. Block diagram of automatic selection of the desired cMUT output signal.

target signal $c(n)$ is defined through coefficients of the MA filter:

$$
\mathbf{c}=\underline{\mathbf{U}} \cdot \mathbf{A}
$$

where the matrix $\underline{\mathbf{U}}$ is defined as $\underline{\mathbf{U}}=\left[\mathbf{u}_{1} \ldots \mathbf{u}_{L}\right]^{T}$ with $\mathbf{u}_{n}=\left[u(n-1) \ldots u\left(n-P_{1}\right)\right]^{T}$ and ${ }^{T}$ the symbol of transposition, the vectors are defined as $\mathbf{c}=[c(1) \ldots c(L)]^{T}, \mathbf{A}=\left[a_{1} \ldots a_{P_{1}}\right]^{T}, P_{1}$ the order of the MA filter and $L$ the sample number of cMUT output signals measured. Note that a low order $\left(P_{1}=2\right)$ similar to a low pass filter is sufficient to extract the linear component.

Finally, the coefficients $\mathbf{A}$ of the MA filter are computed by the least squares method:

$$
\mathbf{A}=\left(\underline{\mathbf{U}}^{T} \underline{\mathbf{U}}\right)^{-1} \underline{\mathbf{U}}^{T} \mathbf{y}
$$

where $\mathbf{y}=[y(1) \ldots y(L)]^{T}$. Note that an easy finite impulse response filter whose cut-off frequencies are adjusted manually would be suitable. However, this adjustment may be difficult, non-optimal and not necessarily automatic. On the other hand, the MA filter can be approximated by a linear finite impulse response filter whose cut-off frequencies are optimally and automatically tuned to eliminate the nonlinear components of the cMUT output.

\section{Automatic Compensation Process}

The next step consists of looping the system by a feedback from the output to the input (Fig. III). This closed loop makes it possible to find the parameters of a nonlinear filter which describes the optimal input $x_{\text {opt }}(n)$ applied to the cMUT, where $n$ is the discrete time. The implementation of this closed loop requires two steps: (i) definition of the criterion to be optimized and (ii) finding the optimal parameter to optimize the cost-function chosen. The advantage of this solution is that it uses the information available in the output signal $y(n)$ to correct the input signal $\hat{x}(n)$ [23].

\section{A. Cost-Function}

The cost-function $J$ is measured on the cMUT output signal $y(n)$. Moreover, it has to be sensitive to modifications of the input signal $\hat{x}(n)$ applied to the cMUT. 


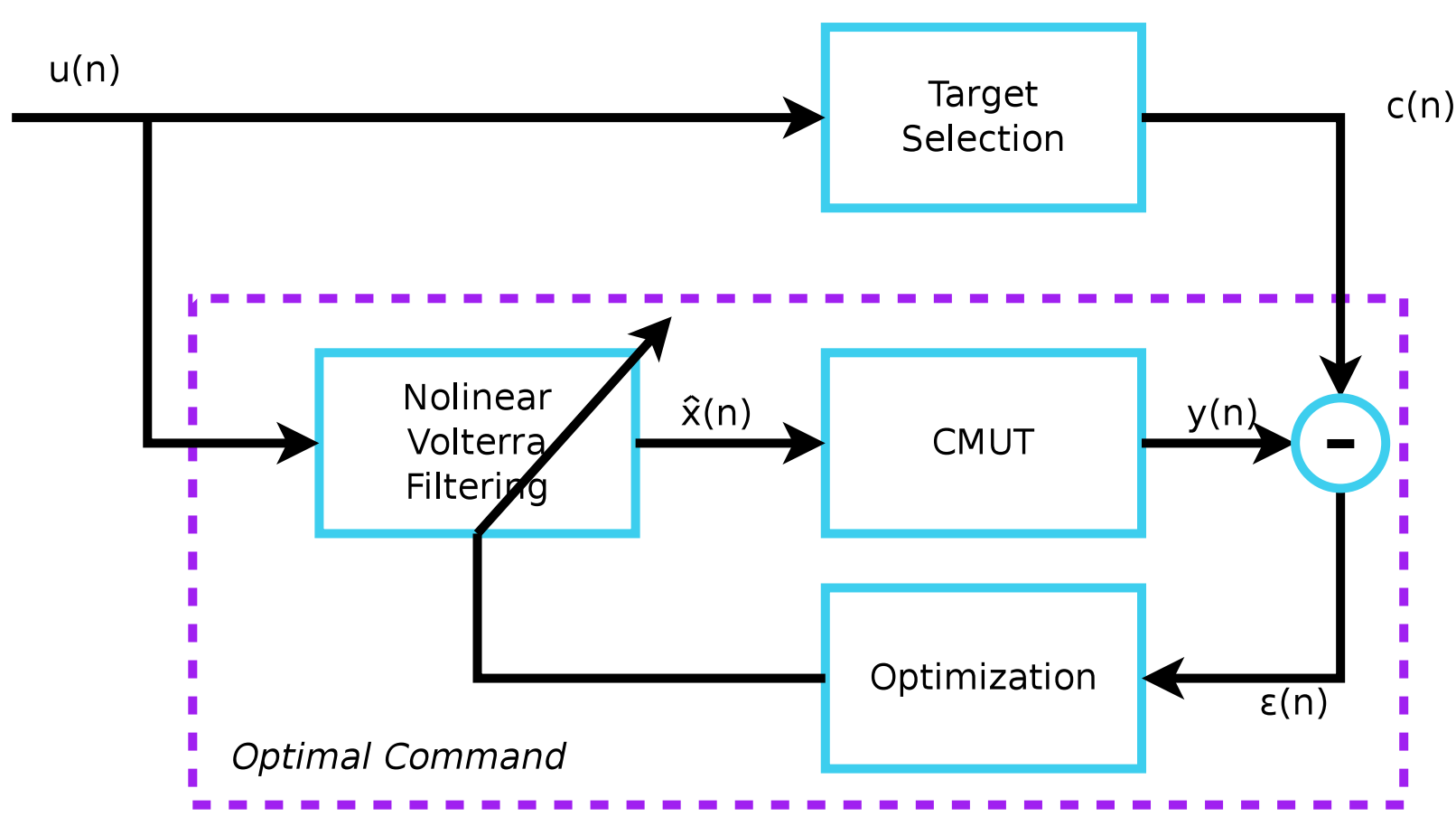

Fig. 2. Block diagram of adaptive system for pre-setting of cMUT.

We therefore compare the measured output $y(n)$ and the target signal $c(n)$ through the time error $\varepsilon(n)$ defined as follows:

$$
\varepsilon(n)=y(n)-c(n) .
$$

At the end of the procedure, i.e. when this error $\varepsilon(n)$ vanishes, the output signal $y(n)$ is similar to the target $c(n)$ which is composed of only the fundamental component. Note that the error $\varepsilon(n)$ is a function of the input signal $\hat{x}(n)$, since the output signal $y(n)$ itself depends on the input signal $\hat{x}(n)$, i.e. $y(n)=y(n, \hat{x}(n))$. Optimization can be achieved by the following equation:

$$
x_{\text {opt }}(n)=\arg \min _{\hat{x}(n)} J\{\varepsilon[y(n, \hat{x}(n)), c(n)]\},
$$

where $\arg \min$ represents the argument when the cost-function $J$ is minimal.

The cost-function $J$ is a function of the error $\varepsilon(n)$. In order to achieve iterative optimization, it becomes the normalized mean squared error $n M S E_{k}$ between the target signal $c(n)$ and the measured output signal $y(n)$, as cost-function $J$ :

$$
n M S E_{k}=10 \cdot \log _{10}\left(\frac{\sum_{i} \varepsilon_{k}^{2}(i)}{\sum_{i} c^{2}(i)}\right),
$$

where $k$ is the iteration. Note that the advantage of the iterative optimization process is to provide a solution which minimizes the error whatever the time $n$. As an illustration, a normalized mean squared error $n M S E$ of $-10 \mathrm{~dB}$ represents an mean error of $10 \%$ between the power of the target signal $c(n)$ and the power of the output signal measured $y(n)$.

\section{B. Nonlinear Filtering of Input Signal}

The second step of the closed loop implementation consists of finding the optimal input signal $x_{\text {opt }}(n)$ which minimizes the normalized mean squared error $n M S E$. For the sake of simplicity, this shape optimization problem is transformed into a parametric optimization problem by tuning the optimal parameters $\mathbf{h}$ of the nonlinear filter which describes the input signal $\hat{x}(n)$ :

$$
x_{\text {opt }}(n)=\arg \min _{\mathbf{h}} n M S E .
$$

Since the harmonic generation in the input signal should make possible the suppression of harmonic components in the output signal, these parameters $\mathbf{h}$ have to generate a signal with different frequency components. Based on the Volterra series for linearization [20], the input signal $\hat{x}(n)$ is transformed into a $P_{2}$-order polynomial series. Put simply, each $P_{2}$-order component of the series corresponds to the input signal $u(n)$ raised to $P_{2}$ and filtered with the unknown coefficients $h_{P_{2}}(n)$. The studies reported by Zhou et al. [15] and Novell et al. [17] were used as reference to fix the order of polynomial expansion. They 
added a signal of frequency $2 f_{0}$ or $3 f_{0}$ to the fundamental component at frequency $f_{0}$ to suppress harmonic components. The solution therefore has to generate harmonic components of second and third order. We chose the Volterra filter of order $P_{2}=3$ described by the parameters $\mathbf{h}$ and the input signal $u(n)$ :

$$
\begin{aligned}
\hat{x}_{k}(n)=\sum_{i=1}^{M} h_{1, k}(i) u(n-i) & +\sum_{i=1}^{M} \sum_{j=i}^{M} h_{2, k}(i, j) u(n-i) u(n-j) \\
& +\sum_{i=1}^{M} \sum_{j=i}^{M} \sum_{l=j}^{M} h_{3, k}(i, j, l) u(n-i) u(n-j) u(n-l),
\end{aligned}
$$

where $M$ is the filter memory. Note that the parameters $\mathbf{h}$ do not depend on the time, which simplifies the problem. The input signal $\hat{x}_{k}(n)$ can also be written in algebraic form:

$$
\hat{\mathbf{x}}_{k}=\underline{\mathbf{V}} \cdot \mathbf{h}_{k}
$$

where

$$
\begin{gathered}
\hat{\mathbf{x}}_{k}=\left[\hat{x}_{k}(M), \hat{x}_{k}(M+1), \ldots, \hat{x}_{k}(L)\right]^{T}, \\
\mathbf{h}_{k}=\left[h_{1, k}(1), h_{1, k}(2), \ldots, h_{1, k}(M), \begin{array}{l}
h_{2, k}(1,1), h_{2, k}(1,2), \ldots, h_{2, k}(M, M), \\
\left.h_{3, k}(1,1,1), h_{3, k}(1,1,2), \ldots, h_{3, k}(M, M, M)\right]^{T},
\end{array}\right. \\
\underline{\mathbf{V}}=\left[\mathbf{v}_{M}, \mathbf{v}_{M+1}, \ldots, \mathbf{v}_{L}\right]^{T},
\end{gathered}
$$

with

$$
\begin{aligned}
\mathbf{v}_{n}=[u(n-1), u(n), \ldots, u(n-M), & u^{2}(n-1), u(n-1) u(n-2), \ldots, u^{2}(n-M), \\
& \left.u^{3}(n-1), u^{2}(n-1) u(n-2), \ldots, u^{3}(n-M)\right]^{T} .
\end{aligned}
$$

However the parameter number $\mathbf{h}$ has to be limited in order to preserve the advantages of the parametric optimization. It is therefore important to choose the memory $M$ correctly. To preserve the advantage of the Volterra series with crossterms (second and third part in eq. 7), the memory has to be greater than 1. However, since the optimization process becomes more difficult when the parameter number increases, it seems advisable to limit the memory $M$ to a low value. A good tradeoff leads to choosing $M=2$, which represents 9 parameters set simultaneously.

Finally, to find the optimal solution to resolve equation 6 , it is only necessary to find the optimal coefficient vector $\mathbf{h}^{\star}$. Note that it is not possible to find an algebraic solution, since the output signal $y(n)$ is a nonlinear transformation of the input signal $\hat{x}(n)$.

\section{Algorithm}

The last step in the implementation of the closed loop is the choice of the algorithm which finds the coefficients $\mathbf{h}$ automatically.

Since we did not have the analytical expression of the normalized mean squared quadratic error $n M S E$ as a function of the parameters $\mathbf{h}$, we needed an algorithm without derivative. Its computation has the advantage of increasing the robustness of the optimization when the computation of the derivative is difficult. One of the most well-known is the Nelder-Mead method [24], [25]. This algorithm (described in Appendix A) is based on the simplex concept which is the generalization of a triangle with $D+1$ vertices $s_{0}, \ldots, s_{D}$ in a dimension space $D$. Note that for our case the space dimension $D$ is 9 , since $\mathbf{h}$ is constituted of 9 parameters. Thus in the space of ten dimensions, the coordinates of a vertex $s_{d}$ are given by the coefficients $\mathbf{h}_{k, d}$.

To initialize the algorithm (i.e. iteration $k=0$ ), an initial simplex is formed from the coefficients $\mathbf{h}_{0,0}$ which make possible the reconstruction of an input signal $\hat{x}_{0}$ identical to the output signal without compensation $y_{0}(n)$. These coefficients are identified by the least mean squares method:

$$
\mathbf{h}_{0,0}=\left(\underline{\mathbf{V}}^{T} \underline{\mathbf{V}}\right)^{-1} \underline{\mathbf{V}}^{T} \mathbf{y}_{\mathbf{0}}
$$

Note that the other vertices $\mathbf{h}_{0, d}$ are computed from the first $\mathbf{h}_{0,0}$ by adding 1 to $d$-th coefficient, as follows:

$$
\underline{\mathbf{H}}_{0}=\left[\mathbf{h}_{k, 0}, \ldots, \mathbf{h}_{k, 0}\right]+\mathbb{I},
$$

where $\underline{\mathbf{H}}_{0}=\left[\mathbf{h}_{k, 0}, \mathbf{h}_{k, 1}, \ldots, \mathbf{h}_{k, D}\right]$ and $\mathbb{I}$ is the identity matrix of ten dimensions.

The initial simplex then moves, expands and contracts through elementary geometric transformation (reflection, expansion, contraction and shrinkage). At each iteration $k$, the method selects the appropriate transformation by using only the value of the normalized mean squared error $n M S E_{k}$. The simplex vertices gradually near the point where the normalized mean squared error $n M S E_{k}$ is minimal. 
Finally, the algorithm stops when the simplex reaches a minimum size [26], [27]:

$$
\frac{1}{\max \left(1,\left\|\mathbf{h}_{k, 0}\right\|\right)} \max _{2 \leqslant i \leqslant D+1}\left\|\mathbf{h}_{k, i}-\mathbf{h}_{k, 0}\right\|<\epsilon,
$$

where $\epsilon=10^{-4}$. In this case, $k$ is the number of iterations required to reach convergence.

To summarize, after transmitting the non-compensated signal $u(n)$ with the desired amplitude, the first output of the transducer $y(n)$ is identified (eq. 9) and makes it possible to create the first simplex. The algorithm (described in Appendix A) is thus initialized and it computes new coefficients $\mathbf{h}$ in order to minimize the normalized mean squared error $n M S E$. From these coefficients, it is possible to construct the optimal input signal $x_{\text {opt }}(n)$.

\section{Application to Medical Linear Array}

To assess the effectiveness of our linearization method, experiments were undertaken using a cMUT-based linear array medical device (Vermon SA, Tours, France). The output signal was the mechanical displacement collected at the front face of the probe. After description of the experimental setup, the results are presented for three situations, i.e. for two low bandwidth excitations and for a high bandwidth excitation.

\section{A. Experimental Setup}

The experimental protocol followed the principle described in [28]: (i) the cMUT was plunged in water, (ii) one element was electrically excited by the compensated signal $\hat{x}_{k}(n)$ and (iii) the mechanical displacement at the centre of the element was collected.

The device under test was made of 64 elements for which the pitch was $200 \mu \mathrm{m}$ and the elevation $5 \mathrm{~mm}$. As shown in Fig. IV-A, the experimental electroacoustic response of one element was measured with a 0.2-mm needle hydrophone (Precision Acoustics, Dorchester, Dorset, UK). The cMUT was designed to work at a central frequency of $4.8 \mathrm{MHz}$ and a fractional bandwidth of $113 \%$ at $-6 \mathrm{~dB}$. Fabrication was by a standard surface micromachining process. Each element was composed of three columns of rectangular cMUTs (dimensions $60 \times 30 \mu \mathrm{m}^{2}$ ). The experimental collapse voltage value was measured with electrical impedance measurements, and a value of $77 \mathrm{~V}$ was determined.

The front face of the probe was covered with a layer of polymer silicone on which a metallized mylar film was stuck to allow laser measurements. To perform experiments and validate the linearization procedure implemented, it was decided to test the efficiency of the method at a frequency where the cMUT shows significant nonlinear behaviour. It is known that the closer the excitation frequency is to the central frequency, the lower the amplitude of nonlinear components [6]. It was shown in reference [29] that when a cMUT works in an elastic regime, even if the excitation frequency is out of the fundamental frequency band, it can provide significant acoustic pressure but with high levels of nonlinear components. Thus, in view of the characteristics of the probe prototype used here, the excitation frequency was fixed at a value close to $1 \mathrm{MHz}$ for all experiments. The excitation amplitude and bias voltage were determined as trade-off between two situations, i.e. i) a low bias voltage value with a high excitation amplitude and ii) a high bias voltage value with a low excitation amplitude. In the first case, the electronic transmitter had to supply at least $100 \mathrm{~V}$ peak-to-peak without creating distortion of the excitation signal at $1 \mathrm{MHz}$ in order to obtain significant output pressure amplitude. In the second case, when the bias voltage and the collapse voltage were too close, amplitude of excitation signal was limited to avoid dynamic membrane collapse, since charging effects could arise and the output pressure amplitude could saturate. Here a bias voltage fixed at $65 \%$ of the static collapse voltage was the trade-off, i.e. $50 \mathrm{~V}$. Note that for these first experiments, the bias voltage value was not considered as a parameter to be optimized because switching the DC voltage applied to the cMUT in real time required designing and adding specific protection circuits that were clearly not within the scope of the study.

The input signal $\hat{x}_{k}(n)$ was delivered by an arbitrary function generator (AFG3102, Tektronix, Beaverton, OR, USA) and then amplified by a power amplifier (150A100B, Amplifier Research, Souderton, PA, USA). The arbitrary waveform generator was linked to a personal computer (PC) through a GPIB bus. The mechanical displacement was collected with a laser interferometer (TEMPO-200, Bossa Nova Tech, Culver City, CA, USA). Note that, since measurements here were performed on the axis of the element, the output of the laser interferometer was always calibrated by combination of Doppler and acousto-optic effects [30]. The output signal $y_{k}(n)$ provided by the laser interferometer was digitized by an oscilloscope (waveRunner 104Xi, LeCroy, Chestnut Ridge, NY, USA) and transferred to the PC. Nonlinear filtering was thus performed by the PC to compute the input signal of the next iterative $k+1$.

Finally, as an illustration and after linearization, the cMUT displacement generated an ultrasound wave in a water tank. This output far-field pressure was measured at $10 \mathrm{~mm}$ from the silicone surface by the same hydrophone.

\section{B. Results}

Several experiments were performed with different target signals in preparation for an encoded imaging context, and a low bandwidth input signal was used first to assess the efficiency of our method. The first target signals were therefore a tone burst centred at $1 \mathrm{MHz}$ with a $-6 \mathrm{~dB}$ relative bandwidth of $45 \%$. A wide bandwidth excitation signal was then used, e.g. a 
(a)

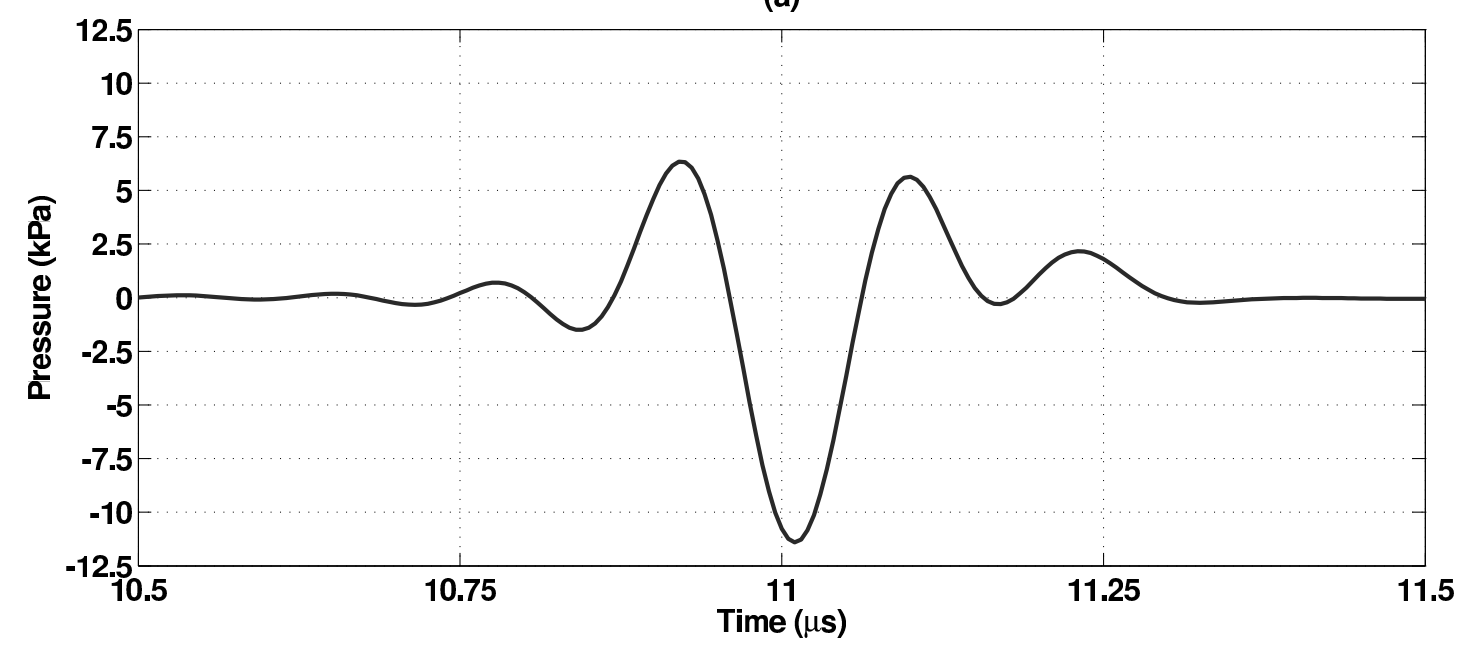

(b)

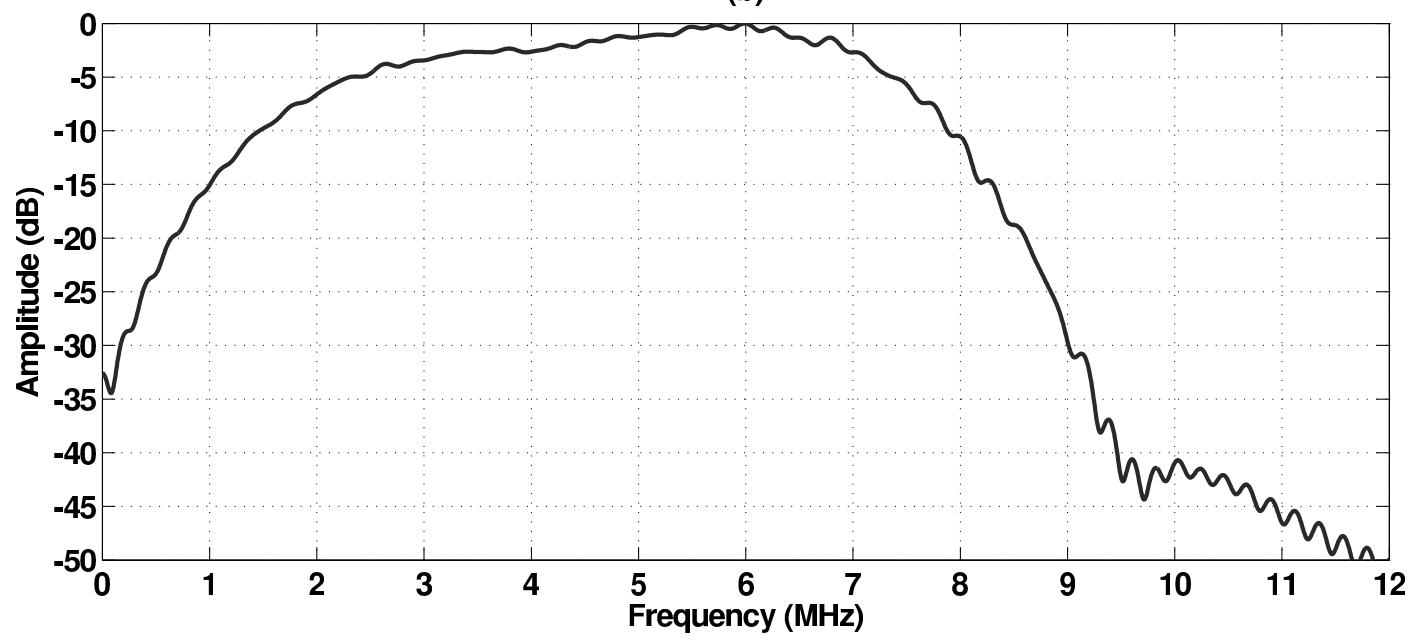

Fig. 3. (a) Experimental electroacoustic response of one element and (b) its respective spectra.

standard frequency modulated chirp. These were characterized by the instantaneous frequency $f_{\text {inst }}$ and the phase $\varphi$, while the modulation amplitude of Gaussian shape was identical for each signal [31]:

$$
u(n)=\exp \left[-2 \alpha\left(\frac{t-t_{0}}{t_{m}}\right)\right] \cos \left(2 \pi\left(f_{0}+\frac{S_{0}}{2} t\right) t+\varphi\right),
$$

where $t=n \cdot f_{s}, t_{0}=t_{m} / 2, t_{m}=5.7 \mu \mathrm{s}, \alpha=3$ and $f_{\text {inst }}=f_{0}+S_{0} / 2 t$. Note that if $S_{0} \neq 0$, then the non-compensated input signal $u(n)$ is a chirp; otherwise the non-compensated input signal $u(n)$ is an apodized sinusoid.

Table IV-B summarizes the three experiments in which the properties of the non-compensated input signal $u(n)\left(f_{\text {inst }}\right.$ and $\varphi)$ were modified.

\begin{tabular}{llll}
\hline \hline & target-signal 1 & target-signal 2 & target-signal 3 \\
\hline \hline$f_{0}$ & $1 \mathrm{MHz}$ & $1 \mathrm{MHz}$ & $1 \mathrm{MHz}$ \\
\hline$S_{0}$ & $0 \mathrm{~Hz} / \mathrm{s}$ & $0 \mathrm{~Hz} / \mathrm{s}$ & $10 \mathrm{GHz} / \mathrm{s}$ \\
\hline phase & 0 & $\pi$ & 0 \\
\hline \hline
\end{tabular}

TABLE I

PRoPERTIES TO DISTINGUiSh THE TARGET SIGNAL FROM THE NON-COMPENSATED SIGNAL $u(n)\left(f_{\text {inst }}\right.$ AND $\varphi$ ) FOR THREE DIFFERENT SITUATIONS.

The first target signal was thus an apodized sinusoid. The second target signal was identical to the first, but in the opposite phase. The last target signal included a frequency modulation to obtain a chirp. 
1) Situation 1: The first column in Table IV-B shows the target properties in the first instance.

Fig. IV-B1a represents the optimal command $x_{\text {opt }}(n)$ and the non-compensated input signal $u(n)$ across the cMUT after amplification. Fig. IV-B1c shows their respective spectra. Note that when the input voltage is positive (with the same sign as the polarisation voltage) the cMUT membranes are stretched, and when the voltage is negative the cMUT membranes are released. The optimal command $x_{\text {opt }}(n)$ has a higher negative amplitude than positive amplitude, which generates harmonic components (second and third order) compared to the non-compensated signal. This asymmetry can be explained by the difference in the behaviour of a cMUT depending on whether the membrane is released or stretched by the electrostatic pressure applied.

Fig. IV-B1b depicts the target signal $c(n)$, the mean displacement when the input signals were the optimal command and the non-compensated input signal $u(n)$. Fig. IV-B1d shows their respective spectra. In the situation with the non-compensated input signal $u(n)$, the mean displacement without compensation had a high second harmonic component and no third harmonic component. This second harmonic component was below $-35 \mathrm{~dB}$ if the input signal was the optimal command $x_{\text {opt }}(n)$. From a time viewpoint, the asymmetry was reduced while the amplitude of displacement was maintained.

As an illustration, Fig. IV-B1e depicts the far-field pressures generated by one element with the optimal command $x_{\text {opt }}(n)$ and with the non-compensated input signal $u(n)$. Fig. IV-B1f shows their respective spectra. The second harmonic was made more visible in the pressure signal by using the non-compensated input signal $u(n)$. The pressure obtained with the optimal command $x_{\text {opt }}(n)$ confirmed the good result measured on the displacement.
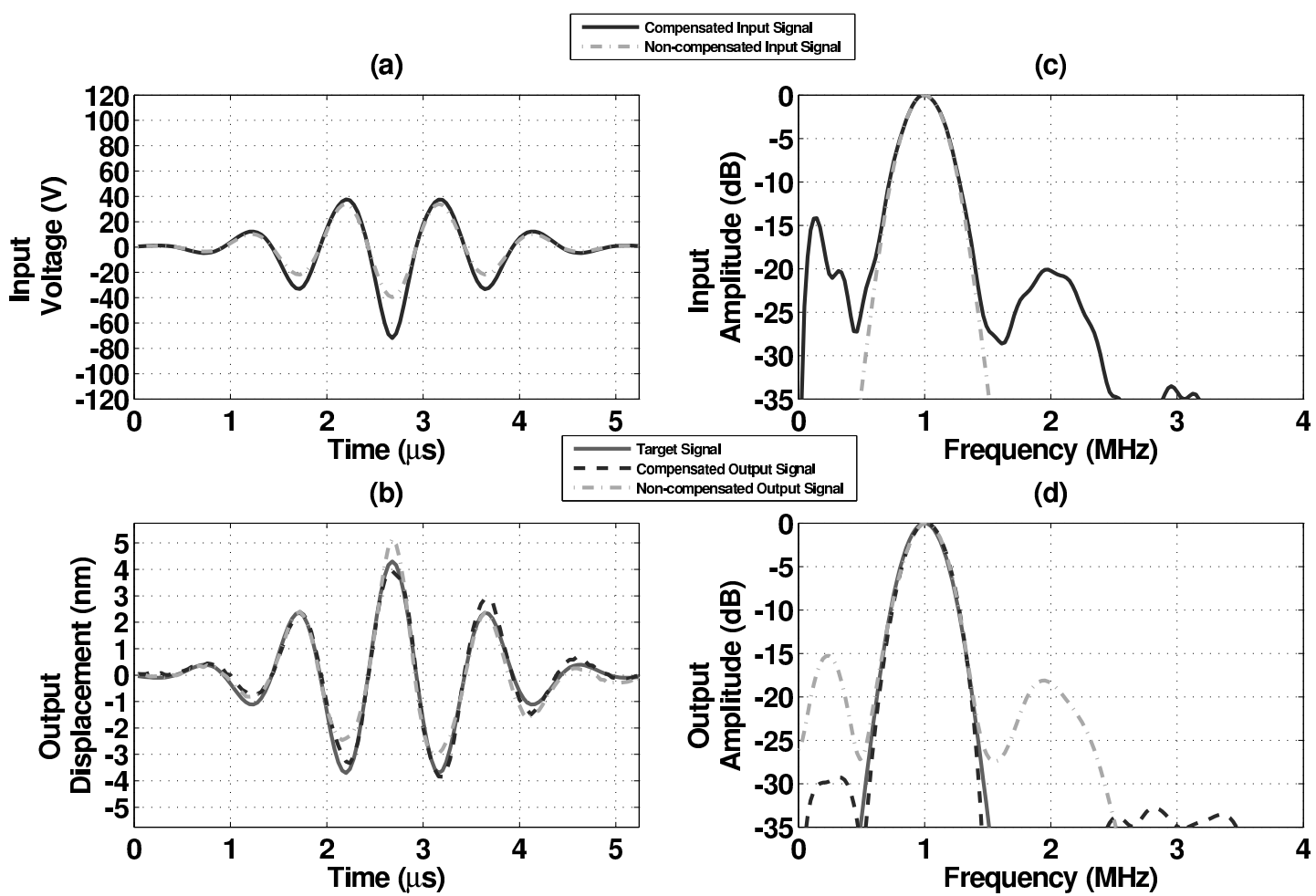

(e)
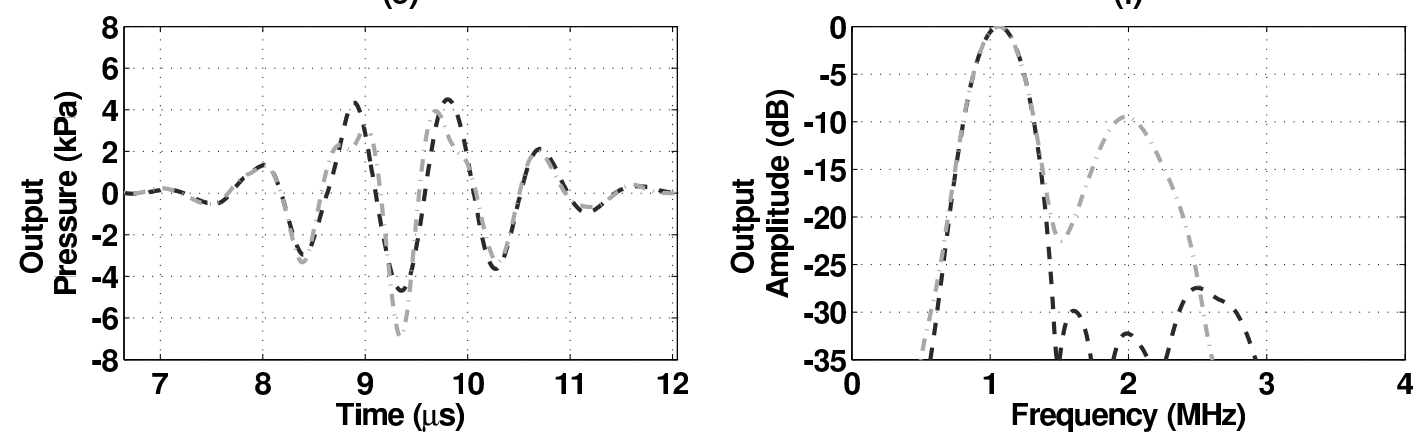

Fig. 4. Compensation of cMUT excitation for a target signal $c(n)$ of sine shape with frequency $f_{0}=1 \mathrm{MHz}$, phase $\varphi=0$ and without frequency modulation $S_{0}=0$ (situation 1). (a) Optimal command $x_{o p t}(n)$ compared to non-compensated input signal $u(n)$ and (c) their respective spectra. (b) Mean displacement with the optimal command $x_{\text {opt }}(n)$ and with the non-compensated input signal $u(n)$ compared to the target signal and (d) their respective spectra. (e) Far-field pressures generated by one element at $10 \mathrm{~mm}$ from the silicone surface using the optimal command $x_{\text {opt }}(n)$ and using the non-compensated input signal $u(n)$ and (f) their respective spectra. 
2) Situation 2: The second column in Table IV-B shows the target properties in the second instance. The target signal is in phase opposition compared to the first situation.

Fig. IV-B2a represents the optimal command $x_{\text {opt }}(n)$ and the non-compensated input signal $u(n)$ across the cMUT after amplication. Fig. IV-B2c shows their respective spectra. The same conclusions can be drawn as in the previous situation. However, it is obvious that the optimal commands of these two situations are different, because the linearization takes into account the different phases of each target signal.

Fig. IV-B2b depicts the target signal $c(n)$, the mean displacement when the input signals were the optimal command and the non-compensated input signal $u(n)$. Fig. IV-B2d shows their respective spectra. The results were similar to those of the first situation. However, the desired waveform imposed a solution where the membranes were more stretched than in the first situation. Only the second harmonic component was below $-30 \mathrm{~dB}$. Nevertheless, because of the constraint of a different phase, the solution was well within the opposite phase. Note that the second harmonic component could reach the same level as the first by decreasing the amplitude of the target signal $c(n)$. However, by successively using the optimal command of the first and second situations with the same amplitudes, it becomes possible to use pulse inversion imaging [9] with cMUTs.

As an illustration, Fig. IV-B2e shows the far-field pressures generated by one element with the optimal command $x_{\text {opt }}(n)$ and with the non-compensated input signal $u(n)$. Fig. IV-B2f shows their respective spectra. The results obtained with the displacement were confirmed by the pressure measurement.
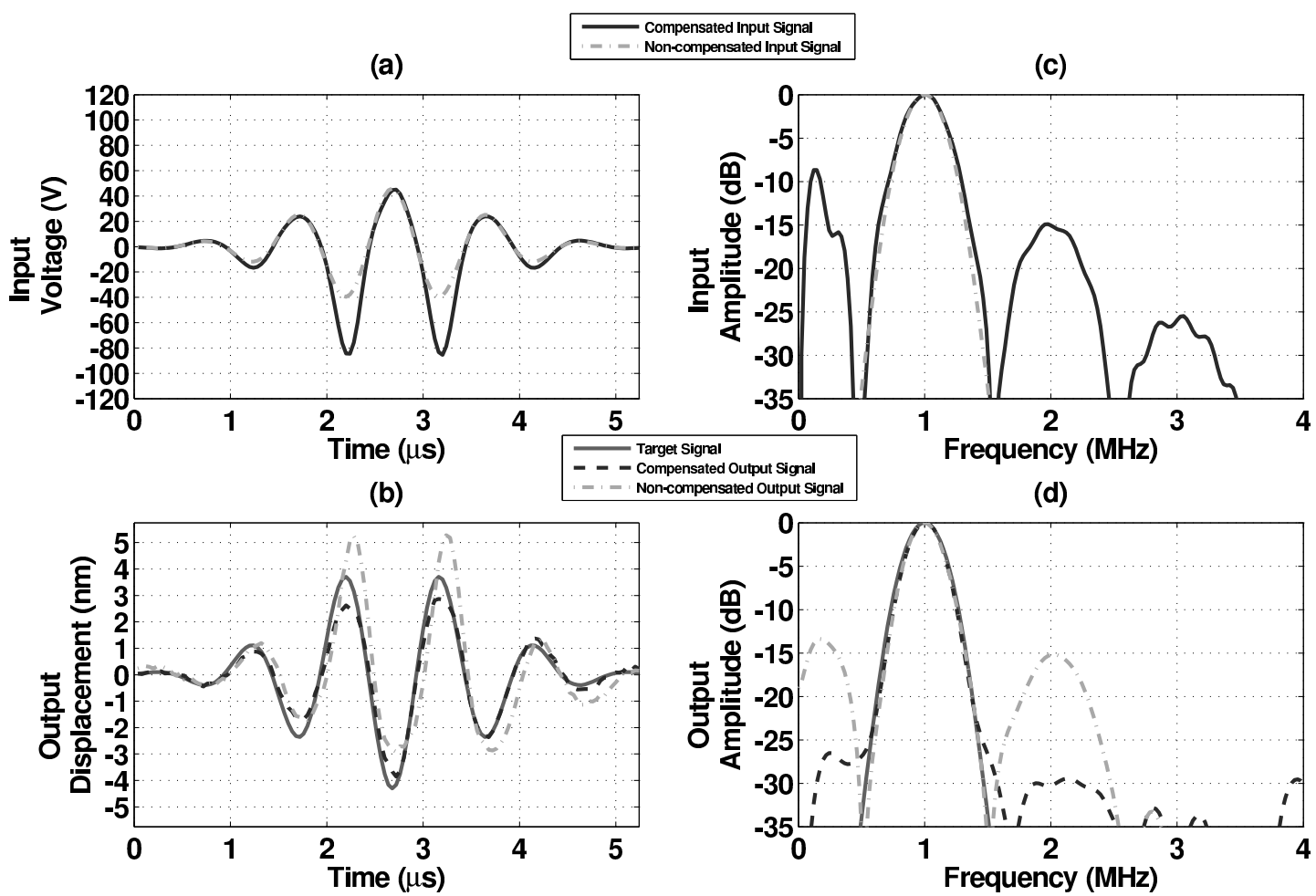

(e)
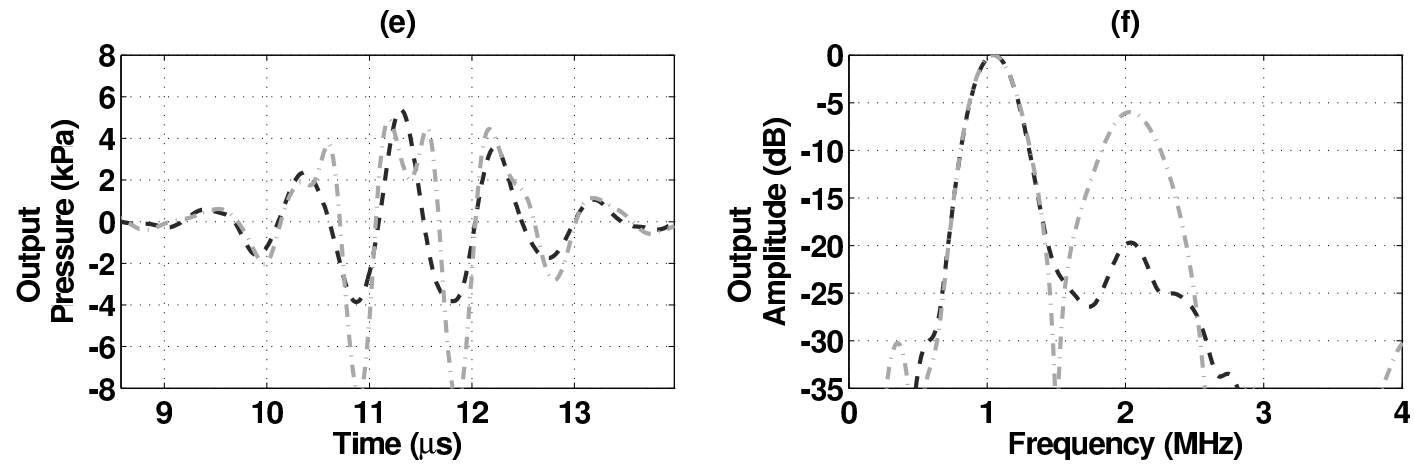

Fig. 5. Compensation of cMUT excitation for a target signal $c(n)$ of sine shape with frequency $f_{0}=1$ MHz, phase $\varphi=\pi$ and without frequency modulation $S_{0}=0$ (situation 2). (a) Optimal command $x_{o p t}(n)$ compared to non-compensated input signal $u(n)$ and (c) their respective spectra. (b) Mean displacement with the optimal command $x_{\text {opt }}(n)$ and with the non-compensated input signal $u(n)$ compared to the target signal and (d) their respective spectra. (e) Far-field pressures generated by one element at $10 \mathrm{~mm}$ from the silicone surface using the optimal command $x_{\text {opt }}(n)$ and using the non-compensated input signal $u(n)$ and (f) their respective spectra. 
3) Situation 3: The third column in Table IV-B shows the target properties in the third instance. The target signal was a chirp, since $S_{0}$ was not equal to zero and therefore introduced a frequency modulation. Note that the chirp makes it possible to observe the linearization for frequencies closer to the central frequency of the cMUT.

Fig. IV-B3a represents the optimal command $x_{\text {opt }}(n)$ and the non-compensated input signal $u(n)$ across the cMUT after amplication. Fig. IV-B3c shows their respective spectra. The same conclusion can be drawn as in the previous situations: the asymmetry of the optimal command $x_{\text {opt }}(n)$ is high to compensate for the cMUT nonlinearities.

Fig. IV-B3b depicts the target signal $c(n)$, the mean displacement when the input signals were the optimal command and the non-compensated input signal $u(n)$. Fig. IV-B3d shows their respective spectra. The results were similar to those of the first two situations. Once again, the method found an optimal command adjusted to the imposed target signal. From a frequency viewpoint, the mean displacement obtained with the optimal command $x_{\text {opt }}(n)$ was close to that of the target signal $c(n)$. However, the error was higher at the beginning of the chirp for low frequencies since their nonlinearities were higher [6], [29], in contrast to the end of the chirp where the frequency is closer to the resonant frequency. Note also that this solution makes it possible to use chirp harmonic imaging [32] with cMUTs.

As an illustration, Fig. IV-B3e shows the pressures generated with the optimal command $x_{\text {opt }}(n)$ and with the noncompensated input signal $u(n)$. Fig. IV-B3f shows their respective spectra. These measurements confirm the results on the displacement. Note that the closest frequencies to the cMUT central frequency were not distorted by the harmonic component when using the optimal command $x_{\text {opt }}(n)$.

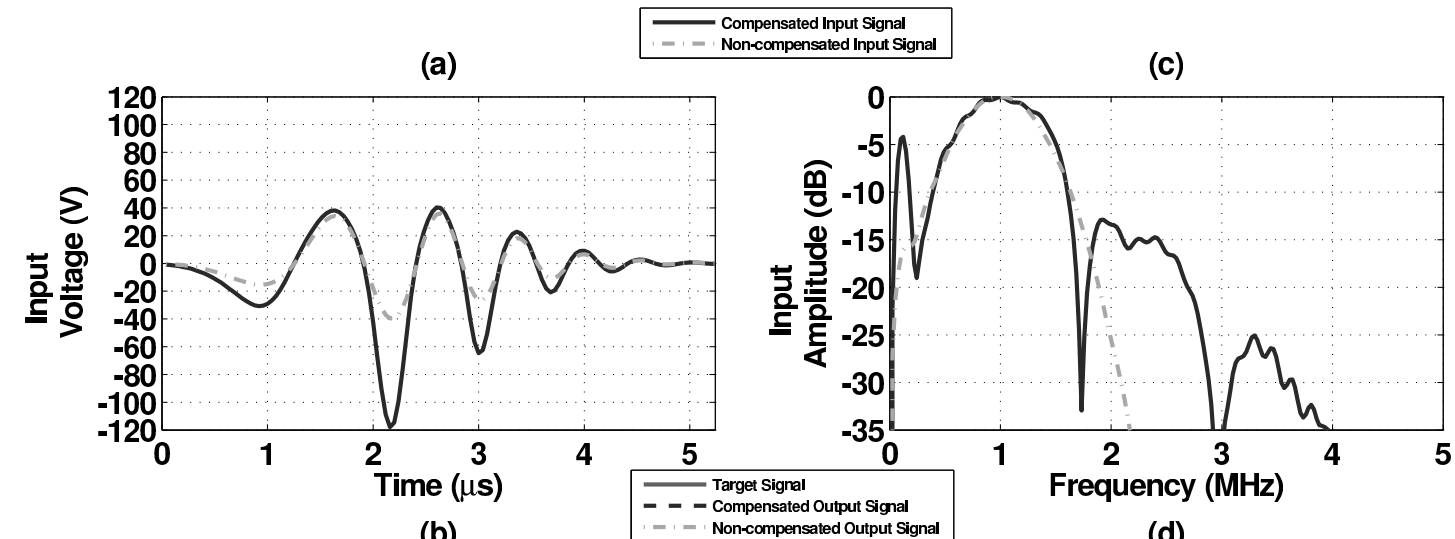

(b)
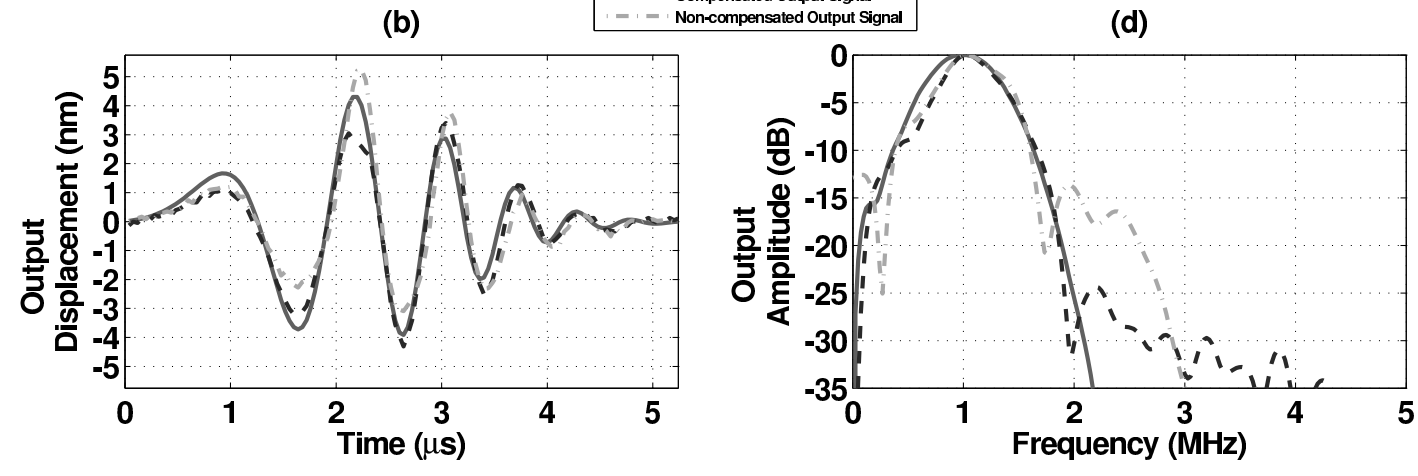

(e)
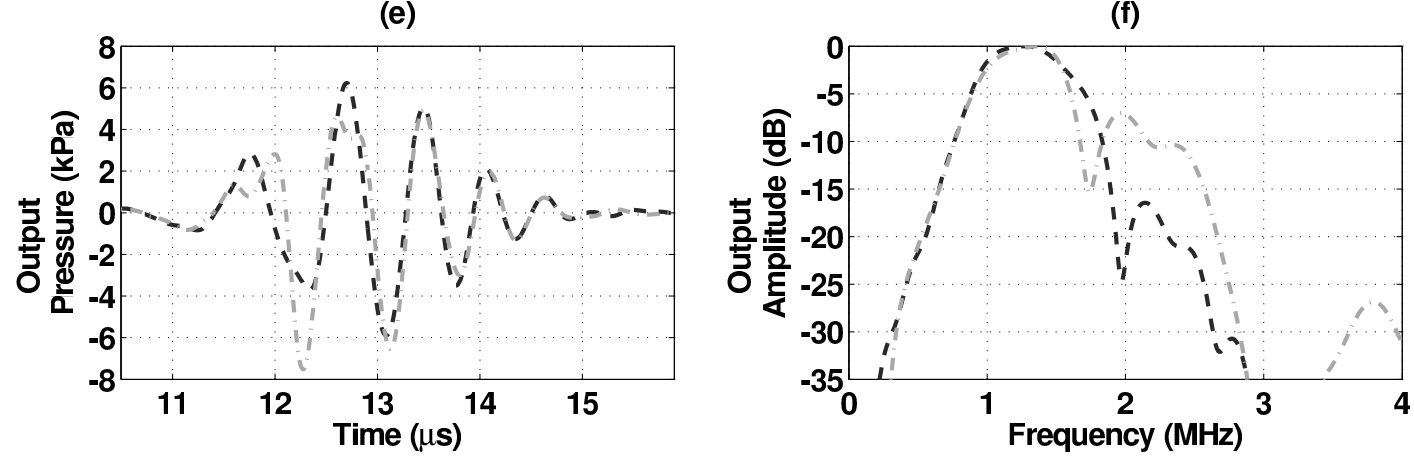

Fig. 6. Compensation of cMUT excitation for a target signal $c(n)$ of sine shape with frequency $f_{0}=1 \mathrm{MHz}$, phase $\varphi=0$ and with frequency modulation $S_{0}=10 \mathrm{GHz} / \mathrm{s}$ (situation 3). (a) Optimal command $x_{\text {opt }}(n)$ compared to non-compensated input signal $u(n)$ and (c) their respective spectra. (b) Mean displacement with the optimal command $x_{\text {opt }}(n)$ and with the non-compensated input signal $u(n)$ compared to the target signal and (d) their respective spectra. (e) Far-field pressures generated by one element at $10 \mathrm{~mm}$ from the silicone surface using the optimal command $x_{\text {opt }}(n)$ and using the non-compensated input signal $u(n)$ and (f) their respective spectra. 
4) Summary of Results: Table IV-B4 summarizes the results of the above three situations by setting out the compensation efficiency (normalized mean squared error $n M S E$, and second harmonic reduction in mean displacement and in far-field pressure) and the number of iterations required to reach convergence.

\begin{tabular}{lllll}
\hline \hline & & target-signal 1 & target-signal 2 & target-signal 3 \\
\hline \hline$n M S E$ & & $-13.2 \mathrm{~dB}$ & $-13.3 \mathrm{~dB}$ & $-12.5 \mathrm{~dB}$ \\
\hline 2nd Harmonic & in Mean Dis- & $-21.2 \mathrm{~dB}$ & $-15.2 \mathrm{~dB}$ & $-6.9 \mathrm{~dB}$ \\
Reduction & placement & & & \\
\cline { 2 - 5 } & $\begin{array}{l}\text { Reduction } \\
\text { in far-field } \\
\text { Pressure }\end{array}$ & $-22.3 \mathrm{~dB}$ & $-14 \mathrm{~dB}$ & $-6.8 \mathrm{~dB}$ \\
& & & \\
\hline $\begin{array}{l}\text { Number of Iterations } \\
\text { before Optimization }\end{array}$ & 186 & 132 & 191 \\
\hline \hline
\end{tabular}

TABLE II

COMPENSATION EFFICIENCY (NORMALIZED MEAN SQUARED ERROR $n M S E$, SECOND HARMONIC REDUCTION IN MEAN DISPLACEMENT AND IN FAR-FIELD PRESSURE) AND THE NUMBER OF ITERATIONS REQUIRED TO REACH CONVERGENCE FOR THE THREE SITUATIONS STUDIED.

Each situation reached a normalized mean squared error $n M S E$ which was equivalent to less than $6 \%$ error between the target power and the output power in fewer than two hundred iterations. The second harmonic component of the output signal was then substantially reduced compared to the output signal without compensation obtained for the non-compensated input signal $u(n)$.

However, this second harmonic reduction was different between the first and the second situations by $6 \mathrm{~dB}$, although the normalized mean squared error $n M S E$ was the same. Indeed, this cost-function of the linearization did not provide a single second harmonic reduction, since it represented the difference between two signals on the full band. Reduction of the harmonic component was thus balanced by an increase in one or several other harmonic components. Note that the second harmonic reduction in far-field pressure showed the same results.

Moreover, this principle was still true for the third situation with the chirp. The second harmonic reduction in the chirp situation was lower than in the previous situations. However, decreasing the normalized mean squared error $n M S E$ amounted to balancing harmonic components for several frequencies in the chirp. Indeed, as described for Fig. 6b, the error between the mean displacement without compensation and the target signal $c(n)$ was higher for the lower frequencies than for the higher frequencies. In order to decrease the normalized mean squared error $n M S E$, it was therefore more important to reduce the high nonlinearities generated by the low frequencies in comparison with the low nonlinearities generated by the high frequencies. However, the fundamental chirp overlapped its the second harmonic. For example, for a chirp frequency beginning at $0.5 \mathrm{MHz}$, the second harmonic occurred at $1 \mathrm{MHz}$ while this frequency was also a fundamental frequency of the chirp. Thus the second harmonic component was mainly due to the high frequencies of the chirp for which the error reduction was less important.

Finally, it is interesting to note that only the first situation can be compared to the existing methods, since the constraints $S_{0}$ and $\varphi$ were zero. In this comparable situation, the effectiveness of our method is similar to that of other methods in terms of second harmonic reduction and waveform. However, we did not observe a significant increase in the third harmonic as in [17].

\section{Discussion AND CONCLUSIONS}

Using this approach, reduction of nonlinearities in the cMUT output was performed using an optimal command. This optimal command was reached automatically by feedback in order to minimize the normalized mean squared quadratic error $n M S E$ between the output signal and a desired signal. Its parametric description made it possible to seek this optimal command automatically by an algorithm. Moreover, this parametric description included a harmonic component of second and third order which could eliminate all the output harmonic components. The compensation method thus made it possible to calibrate the cMUT so that the output signal was equal to the input signal.

Our method is advantageous for two reasons:

- it makes it possible to find the optimal command whatever the desired signal. Although the previous methods limited harmonic reduction to the second harmonic component in the frequency domain (probably more easily identified), some nonlinearity information was lost, since it could be higher order or it could be contained in the phase. By rewriting the problem in the time domain, it is possible to choose any desired signal at the cMUT output without identifying the nonlinearities. However, it obviously requires the target signal to have no undesirable nonlinearities, which is made possible by using a moving average filter. The nonlinearities are minimized by minimizing the error between the target signal and the measured signal. The direct consequence is the possibility of using cMUTs for encoded imaging, e.g. in chirp imaging;

- it is automatic. When the input is set to find the target, the calibration process does not require any information regarding the cMUT properties to seek the optimal command. The feedback makes it possible to use the information contained in 
the cMUT output to find the optimal command. The method should therefore be applied to finding the optimal command for each cMUT (whatever their physical and geometric properties).

The linearization method can be used as a cMUT calibration before cMUT implementation in the ultrasound system. It is based on measurement of the mechanical displacement. Note that we chose to perform the compensation on the cMUT and thus to observe the physical behaviour of the cMUT directly (without any other physical phenomena such as attenuation or diffraction effects

without any other physical phenomenon such as attenuation or diffraction effects). Such measurement can be delicate since the experimental protocol requires a laser interferometer. However, the output measurement can also be a pressure measurement obtained using a hydrophone. Note that it must change the target signal and the instrumentation accordingly. Indeed, identification of the target signal means it is possible to adjust the target signal amplitude while maintaining the measurement linearity at the cMUT output. This principle also applies to the number of elements studied. To make the experimental protocol easier, we used only two. However, it should be possible to use the whole cMUT probe.

To conclude, while existing methods are manual and they might require greater or lesser information about the cMUT used, our method generalizes these methods without these drawbacks. It is possible to seek the optimal command automatically to obtain the cMUT output signal desired. The phase information is taken into account, and this makes the application of encoded imaging for cMUT with broad bandwidths easier. The next step is to test the operational set up with an ultrasound scanner. Such an automatic method should assist the incorporation of cMUTs in ultrasound imaging systems. Moreover, circuit approaches using inductance in series combined with automatic waveform optimization should be the most promising way to increase the emitted pressure field without generating harmonic components for therapeutic applications.

\section{ACKNOWLEDGMENTS}

We thank M. Legros (Vermon SA, Tours, France) for providing the cMUT probe. 


\section{REFERENCES}

[1] M. I. Haller and B. T. Khuri-Yakub, "A surface micromachined electrostatic ultrasonic air transducer," IEEE T. Ultrason. Ferr., vol. 43, no. 1, pp. 1-6, Jan. 1996

[2] A. S. Erguri, Y. Huang, X. Zhuang, O. Oralkan, G. G. Yarahoglu, and B. T. Khuri-Yakub, "Capacitive micromachined ultrasonic transducers: Fabrication technology," IEEE T. Ultrason. Ferr, vol. 52, no. 12, pp. 2242-2258, Dec. 2005.

[3] G. G. Yaralioglu, A. S. Ergun, B. Bayram, E. Haeggstrom, and B. T. Khuri-Yakub, "Calculation and measurement of electromechanical coupling coefficient of capacitive micromachined ultrasonic transducers," IEEE T. Ultrason. Ferr, vol. 50, no. 4, pp. 449-456, Apr. 2003.

[4] G. Caliano, R. Carotenuto, E. Cianci, V. Foglietti, A. Caronti, A. Iula, and M. Pappalardo, "Design, fabrication and characterization of a capacitive micromachined ultrasonic probe for medical imaging," IEEE T. Ultrason. Ferr., vol. 52, no. 12, pp. 2259-2269, Dec. 2005.

[5] B. T. Khuri-Yakub and Ö. Oralkan, "Capacitive micromachined ultrasonic transducers for medical imaging and therapy," J Micromech Microeng, vol. 21, no. 5, p. 054004, May 2011.

[6] A. Lohfink and P.-C. Eccardt, "Linear and nonlinear equivalent circuit modeling of cmuts," IEEE T. Ultrason. Ferr., vol. 52, no. 12, pp. 2163-2172, Dec. 2005.

[7] P. N. Burns, "Instrumentation for contrast echocardiography," Echocardiogr-J Card, vol. 19, no. 3, pp. 241-258, Apr. 2002.

[8] J. G. Lynn, R. L. Zwemer, A. J. Chick, and A. E. Miller, "A new method for the generation and use of focused ultrasound in experimental biology," $J$ Gen Physiol, vol. 26, no. 2, pp. 179-193, Nov. 1942.

[9] D. H. Simpson, C. T. Chin, and P. N. Burns, "Pulse inversion doppler: A new method for detecting nonlinear echoes from microbubble contrast agents," IEEE T. Ultrason. Ferr., vol. 46, no. 2, pp. 372-382, Mar. 1999.

[10] S. H. Wong, M. Kupnik, R. D. Watkins, K. Butts-Pauly, and B. T. Khuri-Yakub, "Capacitive micromachined ultrasonic transducers for therapeutic ultrasound applications," IEEE T. Bio-Med. Eng., vol. 57, no. 1, pp. 114-123, Jan. 2010.

[11] A. Bouakaz, S. Frigstad, F. J. Ten Cate, and N. de Jong, "Super harmonic imaging: A new imaging technique for improved contrast detection," Ultrasound Med Biol, vol. 28, no. 1, pp. 59-68, Jan. 2002.

[12] H. K. Oguz, S. Olcum, M. N. Senlik, V. Tas, A. Atalar, and H. Koymen, "Nonlinear modeling of an immersed transmitting capacitive micromachined ultrasonic transducer for harmonic balance analysis," IEEE T. Ultrason. Ferr., vol. 57, no. 2, pp. 438-447, Feb. 2010.

[13] S. Satir and F. Degertekin, "Harmonic reduction in capacitive micromachined ultrasonic transducers by gap feedback linearization," IEEE T. Ultrason. Ferr., vol. 59, no. 1, pp. $50-59$, Jan. 2012.

[14] J. I. Seeger and S. B. Crary, "Stabilization of electrostatically actuated mechanical devices," in Int. Conf. Solid State Sensors Actuators, vol. 2, Chicago, USA, Jun. 1997, pp. 1133-1136.

[15] S. Zhou, P. Reynolds, and J. Hossack, "Precompensated excitation waveforms to suppress harmonic generation in mems electrostatic transducers," IEEE T. Ultrason. Ferr., vol. 51, no. 11, pp. 1564-1574, Nov. 2004.

[16] S. Zhou and J. A. Hossack, "Reducing inter-element acoustic crosstalk in capacitive micromachined ultrasound transducers," IEEE T. Ultrason. Ferr. vol. 54, no. 6, pp. $1217-1228$, Jun. 2007.

[17] A. Novell, M. Legros, N. Felix, and A. Bouakaz, "Exploitation of capacitive micromachined transducers for nonlinear ultrasound imaging," IEEE T. Ultrason. Ferr., vol. 56, no. 12, pp. 2733-2743, Dec. 2009.

[18] A. Novell, J.-M. Escoffre, and A. Bouakaz, "Second harmonic and subharmonic for non-linear wideband contrast imaging using a capacitive micromachined ultrasonic transducer array," Ultrasound Med Biol, 2013.

[19] S. Ménigot and J.-M. Girault, "Optimization of contrast-to-tissue ratio by adaptation of transmitted ternary signal in ultrasound pulse inversion imaging," Computational and Mathematical Methods in Medicine, vol. 2013, p. 6, 2013.

[20] V. J. Mathews and G. L. Sicuranza, Polynomial Signal Processing. New York, NY, USA: Wiley - Interscience, 2000.

[21] W. A. Frank, "An efficient approximation to the quadratic volterra filter and its application in real-time loudspeaker linearization," Signal Processing, vol. 45, no. 1, pp. 97-113, Jul. 1995.

[22] S. D. Senturia, Microsystem Design. Heidelberg, Germany: Springer, Nov. 2000.

[23] I. D. Landau, R. Lozano, M. M'Saad, and A. Karimi, Adaptive Control: Algorithms, Analysis and Applications, 2nd ed. London, UK: Springer, Jun. 2011.

[24] J. A. Nelder and R. Mead, "A simplex method for function minimization," The Computer Journal, vol. 7, no. 4, pp. 308-313, Jan. 1965.

[25] J. C. Lagarias, J. A. Reeds, M. H. Wright, and P. E. Wright, "Convergence properties of the nelder-mead simplex method in low dimensions," SIAM Journal of Optimization, vol. 9, no. 1, pp. 112-147, Jul. 1998.

[26] J. Dennis and D. Woods, "Optimization on microcomputers: The nelder-mead simplex algorithm," in New Computing Environments: Microcomputers in Large-Scale Computing. SIAM Philadelphia, 1987, pp. 116-122.

[27] J. J. Tomick, "On convergence of the nelder-mead simplex algorithm for unconstrained stochastic optimization," Ph.D. dissertation, Pennsylvania State University, State College, PA, USA, May 1995.

[28] D. Certon, O. B. Matar, J. Guyonvarch, N. Felix, and F. Patat, "Characterization of transducer arrays by laser interferometry: Influence of acousto-optic interactions on displacement measurements in water," in Proc. IEEE Ultrason. Symp., vol. 2, Atlanta, GA, USA, Oct. 2001, pp. 1065-1068.

[29] N. Sénégond, A. Boulmé, C. Plag, F. Teston, and D. Certon, "Fast time-domain modeling of fluid-coupled cmut cells: from the single cell to the 1-D linear array element," IEEE Transactions on Ultrasonics Ferroelectrics and Frequency Control, vol. 60, no. 7, pp. 1505-1518, Jul. 2013.

[30] D. Royer and O. Casula, "Quantitative imaging of transient acoustic fields by optical heterodyne interferometry," in Proceeding IEEE Ultrasonic Symposium, vol. 2, Cannes, France, Nov. 1994, pp. 1153-1162.

[31] J. E. Wilhjelm, "Bandwidth expressions of gaussian weighted chirp," Electron Lett, vol. 29, no. 25, pp. 2161-2162, Dec. 1993.

[32] J. M. G. Borsboom, C. T. Chin, A. Bouakaz, M. Versluis, and N. de Jong, "Harmonic chirp imaging method for ultrasound contrast agent," IEEE T. Ultrason. Ferr., vol. 52, no. 2, pp. 241-249, Feb. 2005. 


\section{APPENDIX}

Result: Find the optimal coefficient vector $\mathbf{h}^{\star}$

begin

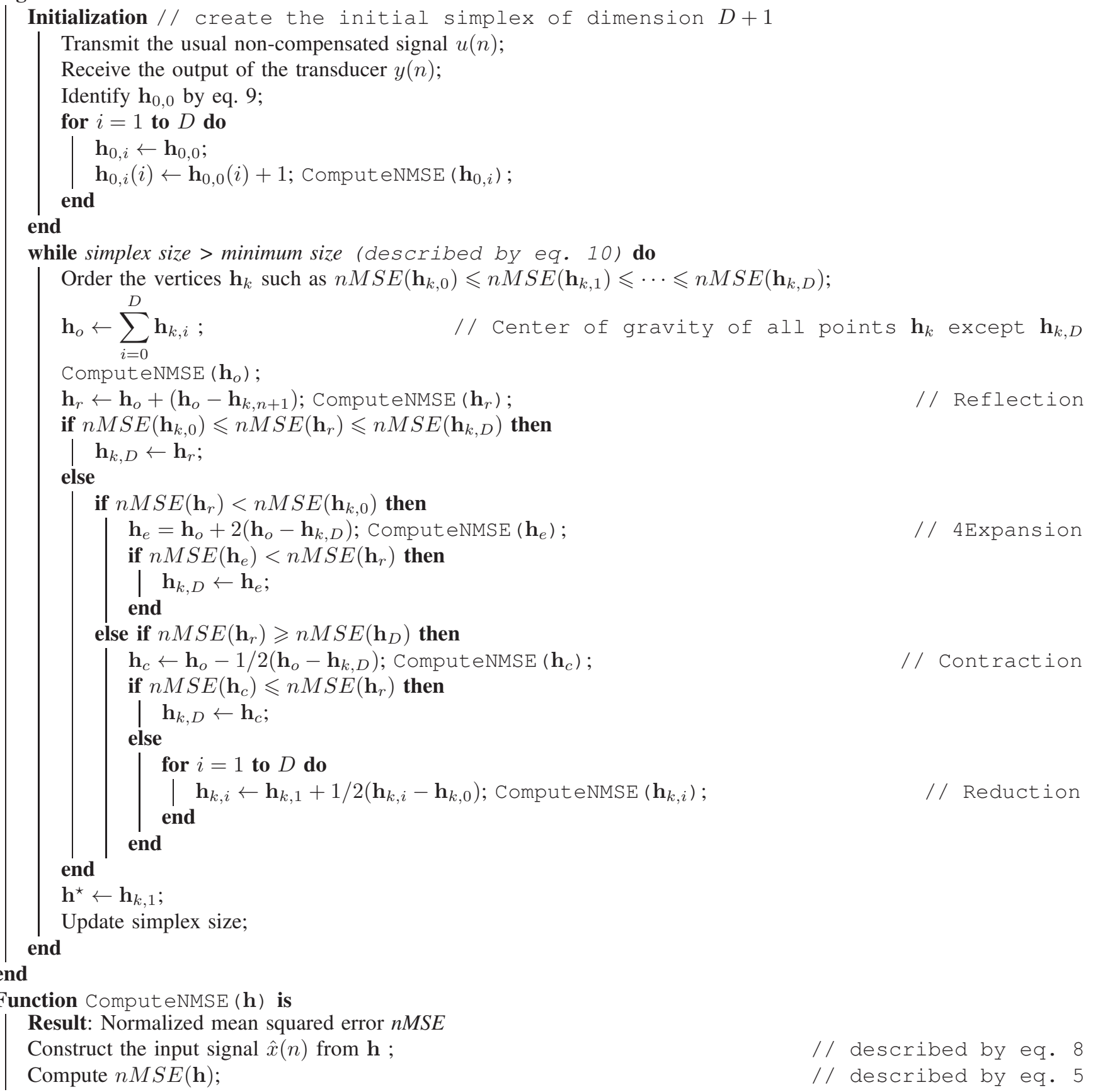
end

Algorithm 1: Pseudo-code of the minimization of the normalized mean squared error by the Nelder-Mead method 


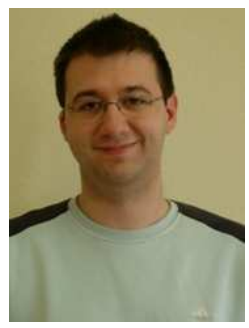

Sébastien Ménigot was born in France in 1985. He received his master's degree in medical imaging technology from the François Rabelais University of Tours, France, in 2008. He obtained his Ph.D. degree in signal processing for medical ultrasound imaging from the François Rabelais University of Tours in 2011. He is also a teaching assistant in electronics and computer science at the Institut of Technology of Ville d'Avray, Université Paris Ouest Nanterre La Défense, France. His research focuses on optimal command applied to ultrasound imaging systems.

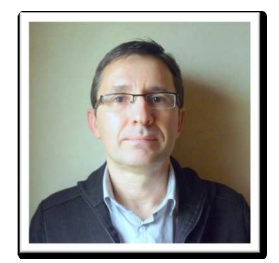

Dominique Certon received an M.Sc. degree in signal processing and electrical engineering in 1991 from the University of Orléans, France. In 1994, he received a Ph.D. degree from the University of Tours, France. Finally, he obtained his Ha- bilitation à diriger les recherches (HDR) in applied acoustics and materials engineering from the François Rabelais University of Tours in 2010. He is an associate professor in telecommunication sys- tems at the University of Tours. His research interests are in ultrasound probes for medical imaging based on capacitive micromachined transducers and piezoelectric technology. He is author or coauthor of more than 50 communications and publications. He is a member of the French Society of Acoustics.

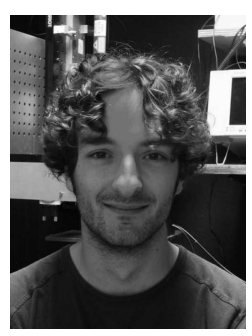

Dominique Gross was born in Strasbourg, France, in 1987. He received his engineering degree in 2010 from the École Nationale Supérieure d'Ingénieurs du Mans in acoustics and vibration. He starts a Ph.D. degree in medical acoustics first in the INSERM UMRS U930 Imaging and Brain Laboratory in 2011 and continues in the CNRS UMR 7347 GREMAN Laboratory since 2012. He is currently focused on the modeling, the design and the characterization of capacitive micromachined ultrasonic transducers (cMUTs) dedicated to medical imaging and therapeutic applications.

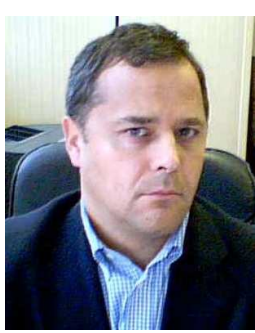

Jean-Marc Girault (S'98-M'00) received his master's degree in signal processing and biological and medical imaging from the University of Angers, France, in 1996. He obtained his Ph.D. degree in signal processing in medical ultrasound imaging from the François rabelais University of Tours, France, in 1999. Finally, he obtained his qualification for research supervision in signal processing in medical ultrasound imaging from the François-Rabelais University of Tours, in 2010. He has been a lecturer in signal processing at the University of Tours since 2001. His principle results achieved were in nonlinear signal analysis (complexity analysis with recurrence, entropies, symmetries and multifractals) and processing (modelling, identification) and in ultrasound imaging. Most important findings were in optimal command (deterministic and stochastic) and blind deconvolution in ultrasound imaging, in subultra-harmonics modelling and identification and in Doppler detectors and estimators (rhythm, velocity, energy) applied for the studies involving microemboli and distressed foetus. He has written more than 90 scientific communications on medical ultrasound imaging and signal processing. Since 2005, he has been the responsible of the master's degree in medical imaging at the François-Rabelais University of Tours. At the same time, he coordinates the "Signal-Imaging" group in the team "Imaging and Ultrasound" in the research unit "Imaging and Brain" UMR U930, Tours, France. 